ARTICLE

Received 25 Apr 2016 | Accepted 20 Jan 2017 | Published 10 Mar 2017

DOI: $10.1038 /$ ncomms14688

OPEN

\title{
EGFR/ARF6 regulation of Hh signalling stimulates oncogenic Ras tumour overgrowth
}

\author{
Chiswili Chabu', Da-Ming $\mathrm{Li}^{1} \&$ Tian $\mathrm{Xu}^{1,2}$
}

Multiple signalling events interact in cancer cells. Oncogenic Ras cooperates with Egfr, which cannot be explained by the canonical signalling paradigm. In turn, Egfr cooperates with Hedgehog signalling. How oncogenic Ras elicits and integrates Egfr and Hedgehog signals to drive overgrowth remains unclear. Using a Drosophila tumour model, we show that Egfr cooperates with oncogenic Ras via Arf6, which functions as a novel regulator of Hh signalling. Oncogenic Ras induces the expression of Egfr ligands. Egfr then signals through Arf6, which regulates $\mathrm{Hh}$ transport to promote $\mathrm{Hh}$ signalling. Blocking any step of this signalling cascade inhibits Hh signalling and correspondingly suppresses the growth of both, fly and human cancer cells harbouring oncogenic Ras mutations. These findings highlight a non-canonical Egfr signalling mechanism, centered on Arf6 as a novel regulator of $\mathrm{Hh}$ signalling. This explains both, the puzzling requirement of Egfr in oncogenic Ras-mediated overgrowth and the cooperation between Egfr and Hedgehog.

\footnotetext{
${ }^{1}$ Department of Genetics, Howard Hughes Medical Institute, Yale University School of Medicine, Boyer Center for Molecular Medicine, 295 Congress Avenue, New Haven, Connecticut 06536, USA. ${ }^{2}$ Children's Hospital \& Institute of Developmental Biology and Molecular Medicine, Fudan University, Shanghai 20043, China. Correspondence and requests for materials should be addressed to T.X. (email: tian.xu@yale.edu).
} 
A ctivating mutations of the Ras gene are highly prevalent in human cancers and give rise to some of the most aggressive tumours ${ }^{1}$. The molecular mechanisms governing oncogenic Ras-driven cancers are complex and involve interacting signalling pathways ${ }^{1,2}$. Paradoxically, oncogenic Ras cooperates with Egfr in cancers $^{3-6}$. EGFR ligands bind to and activate Egfr, which recruits docking proteins via its cytoplasmic domain. Docking proteins (such as the downstream of receptor kinases or drk) activate the guanine exchange factor Son of sevenless (Sos), which converts Ras from an inactive (GDP-bound) to an active (GTP-bound) state and leads to the MAPK signalling cascade and activation of downstream target molecules ${ }^{7,8}$. It is surprising that the action of an activated downstream oncogenic component still requires its upstream receptor.

On the other hand, Egfr has been shown to cooperate with Hedgehog ( $\mathrm{Hh}$ ) signalling, another oncogenic pathway, to drive basal cell carcinoma and melanomas ${ }^{9-12}$. Hh signalling is initiated by the interaction of $\mathrm{Hh}$ protein with its receptor Patched. Endocytosis and intracellular transport of the receptorligand complex modulate $\mathrm{Hh}$ signalling levels ${ }^{13}$. How oncogenic Ras, Egfr and Hh signalling are integrated to concertedly drive tumour overgrowth remains unclear. Animal models expand our understanding of oncogenic Ras signalling.

Using a fly tumour model of oncogenic Ras we have identified Egfr as a positive regulator of oncogenic Ras-mediated overgrowth. Our characterization of Egfr's role in oncogenic Ras-mediated overgrowth led the finding that oncogenic Ras signalling stimulates the expression of the Egfr ligand spitz (spi) to recruit Egfr signalling and achieve tumour overgrowth. Egfr promotes tumour overgrowth independent of the canonical the Sos/Ras signalling, instead it acts via the ADP-Ribosylation Factor 6 (Arf6). Arf6 belongs to a family of highly conserved small Ras-related GTP-binding proteins and is largely known for its role in regulating endocytosis, vesicle transport and secretion ${ }^{14-22}$. We investigated a role for Arf6 in oncogenic Ras tumour overgrowth and found that Egfr promotes Arf6 to interact with $\mathrm{Hh}$. This interaction allows Arf6 to control Hh cellular trafficking and promote $\mathrm{Hh}$ signalling. Consistent with this, blocking Egfr or Arf6 suppresses Hh signalling and inhibits the growth of either fly or human cancer cells harbouring oncogenic Ras. Altogether, our data delineate a non-canonical Egfr signalling mechanism in which Arf6 acts as a novel regulator of Hh signalling. This explains the puzzling requirement of Egfr in oncogenic Ras-mediated overgrowth and the oncogenic cooperation between Egfr and Hh signalling.

\section{Results}

Egfr ${ }^{-}$suppresses $\operatorname{Ras}^{V 12}$ tumours by inhibiting cell proliferation. Mosaic expression of oncogenic Ras $\left(\operatorname{Ras}^{V 12}\right)$ gives rise to hyperplastic tumours in Drosophila tissues ${ }^{23,24}$. These tumours can be GFP-labelled and a measure of the overgrowth phenotype can be readily obtained by examining the size and the fluorescence intensity of clones in dissected eye-antenna imaginal discs from third-instar animals ${ }^{25,26}$ (Fig. 1a,b,f and g). We searched for mutations that suppress Ras ${ }^{V 12}$ tumour overgrowth $^{26}$ and identified a null Egfr mutation EgfrCo (ref. 27), hereafter referred to as Egfr-. We generated clones of cells harbouring the Egfr- mutation or expressing Ras ${ }^{V 12}$ in the presence of the Egfr-mutation and scored clones size. Consistent with EGFR's known role in controlling cell survival and growth ${ }^{28}$, Egfr- mutant cells yielded small clones (Fig. 1a,c,f and h). We found that Egfr- suppressed Ras ${ }^{V 12}$ tumour overgrowth (Fig. 1a,b,d,f,g and i; quantified in Fig. 2j). A dominant-negative version of Egfr (lacking its cytoplasmic tail) ${ }^{29}$ produced a similar effect (Fig. 1e,j). Thus oncogenic Ras-mediated overgrowth requires the function of the upstream receptor Egfr.

We examined how Egfr cooperates with oncogenic Ras. Egfr could exert this tumour-promoting effect by regulating apoptosis and/or cell proliferation. We examined cell death in Ras ${ }^{V 12}$ or Ras ${ }^{V 12}$, Egfr- double mutant clones using terminal deoxynucleotidyl transferase dUTP nick end-labelling (TUNEL) assays. We did not detect ectopic cell death inside Ras ${ }^{V 12}$, Egfr- double mutant clones compared with Ras ${ }^{V 12}$ clones (Fig. $1 \mathrm{k}-\mathrm{n}$ ). Instead, Egfr- reduced the cell proliferation potential of $\mathrm{Ras}^{\mathrm{V} 12}$ cells, as indicated by the decrease in the percentage of phospho-histone3 positive cells in Ras ${ }^{V 12}$, Egfr- double mutant clones compared with $\operatorname{Ras}^{V 12}$ clones (Fig. 1o,q versus p,r; quantified in 1s). Thus, Egfr fosters oncogenic Ras-mediated tumour overgrowth by promoting cell proliferation.

Ras $^{V 12}$ activates spitz to promote non-canonical Egfr signalling. Oncogenic Ras could recruit Egfr's function by upregulating Egfr or its ligands. Oncogenic activation of the MAPK pathway has been shown to trigger the overexpression of Egfr and autocrine activation of Egfr by transforming growth factor alpha (TGF- $\alpha$ ) family of Egfr ligands in cancer cells ${ }^{4,6}$. Drosophila has two TGF- $\alpha$ homologs, Gurken (Grk) and spitz (Spi) ${ }^{30,31}$. The expression and function of Grk are restricted to the germline in embryos, while Spi is broadly expressed and potently activates Egfr throughout all stages of development ${ }^{30,32}$. While we did not detect any change in Egfr protein levels in $R_{a s}{ }^{\dot{V} 12}$ clones (Fig. 2a), an antibody against the extra cellular domain of Spi showed elevated spi protein levels in and around Ras ${ }^{V 12}$ clones (Fig. 2b and b'). This argues that $\operatorname{Ras}^{V 12}$ cells upregulate Spi. Consistent with this, quantitative polymerase chain reaction analyses revealed that Ras $^{V 12}$ transcriptionally stimulates Spi (Fig. 2k). Next, we assessed the functional significance of Spis upregulation on tumour overgrowth. We introduced a null Spi mutation ( $\left.s p i^{-}\right)$in $\mathrm{Ras}^{\mathrm{V} 12}$ cells and scored clone sizes compared with Ras ${ }^{V 12}$ clones. Similar to Egfr-, the spimutation produced small clones and suppressed the overgrowth phenotype of Ras ${ }^{V 12}$ (Fig. 2c,d; quantified in j, and Supplementary Fig. 1d,g,j,m). In addition, we examined the effect of knocking down Star, which is required for Spi secretion and its growth control function (Supplementary Fig. 1a,c, and ref. 33). This also suppressed Ras ${ }^{V 12}$-mediated tumour overgrowth (Fig. 2c,e). Moreover, we took advantage of a conditional ligand-bindingdefective Egfr allele, Egfr $r^{\text {tsla }}$ (ref. 34). We examined the effect of this mutation on $\operatorname{Ras}^{V 12}$ tumour overgrowth at restrictive conditions and found that it also suppressed overgrowth (Fig. 2c,f, and Supplementary Fig. 1d,g,k,n). Collectively, these data argue that oncogenic Ras stimulates spi, which in turn triggers Egfr to increase tumour growth.

Egfr could contribute to oncogenic Ras-mediated overgrowth by augmenting canonical Egfr signalling levels via $\mathrm{Ras}^{35}$. Alternatively, Egfr signalling could exert its growth promoting effect via a previously uncharacterized mechanism. To distinguish between these two possibilities, we first tested whether knocking down Sos has an effect on Ras V12 tumour overgrowth. While the expression of Sos-RNAi caused a small eye phenotype in adult animals, consistent with Egfr/Ras signalling inhibition (Supplementary Fig. 1a,b,d,g,l and o), it showed no effect on oncogenic Ras ${ }^{V 12}$ tumour overgrowth (Fig. 2c versus g). Similarly, direct inactivation of wild-type Ras using a null allele ( $\mathrm{ras}^{\mathrm{C} 40 \mathrm{~A}}$ ) (ref. 36), produced small clones (Fig. 2h) but failed to suppress the Ras ${ }^{V 12}$ tumour overgrowth phenotype (Fig. 2c versus i). Taken together, the above data indicate that ligand-mediated Egfr function promotes oncogenic Ras tumour overgrowth independent of canonical Egfr signalling. 

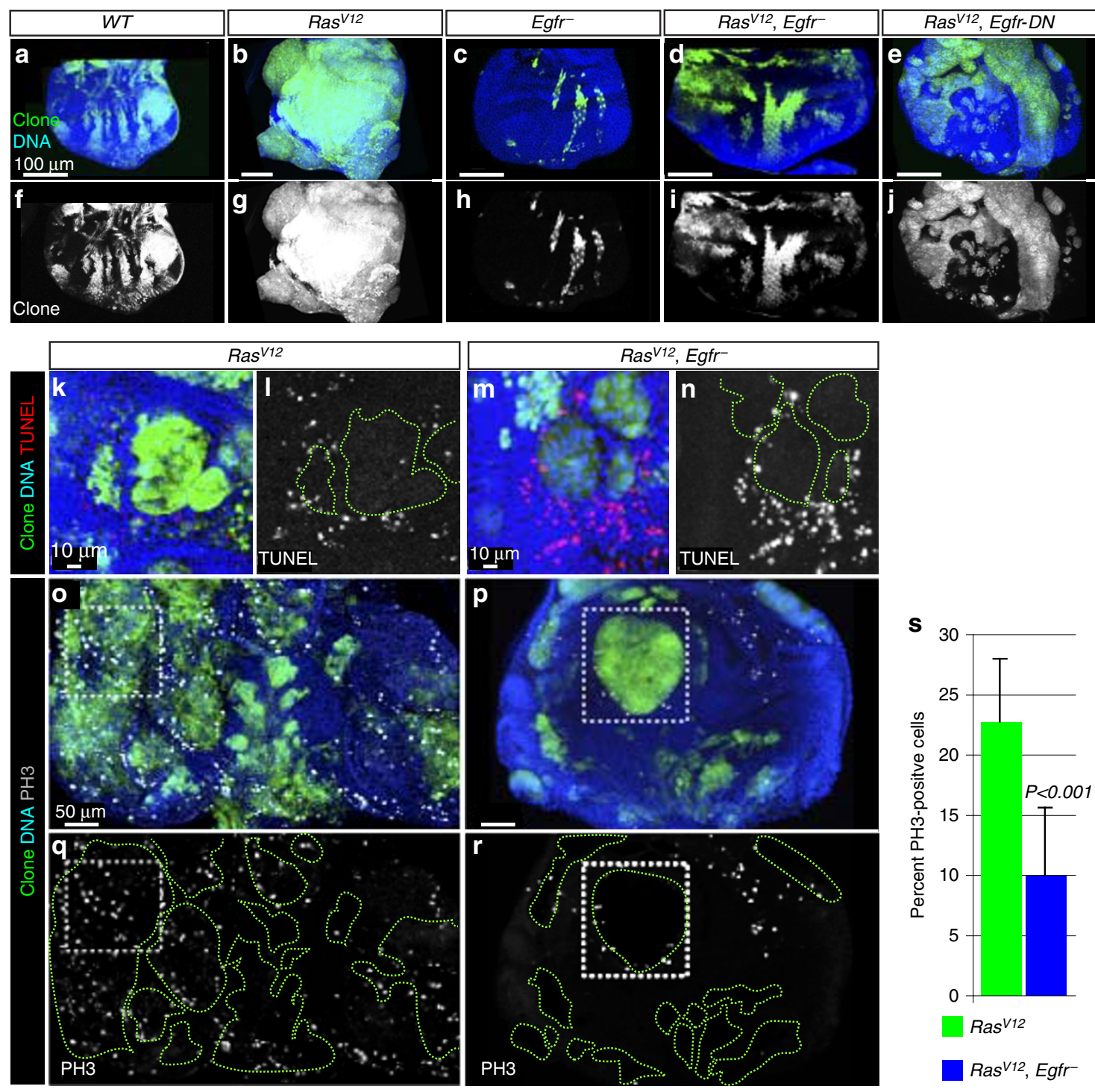

Figure 1 | Egfr ${ }^{-}$suppresses Ras $^{\mathbf{V 1 2}}$ tumour overgrowth by inhibiting cell proliferation. (a-j) Images of eye discs containing GFP-labelled wild-type or $\mathrm{Ras}^{\mathrm{V} 12}$ or Egfr ${ }^{-}$single mutant or Ras ${ }^{\mathrm{V} 12}$, Egfr ${ }^{-}$or Ras ${ }^{\mathrm{V} 12}$, Egfr-DN double mutant clones dissected from wondering third-instar animals raised at $25^{\circ} \mathrm{C}$. Images represent a projection of the top $10 \mu \mathrm{m}$ for each genotype. Ras ${ }^{V 12}$ clones (b) overgrow to form large contiguous tumours compared with control clones (a). The Egfr ${ }^{-}$mutation yields small clones (c) and suppresses the growth of Ras ${ }^{V 12}$ clones (d). Expression of a dominant version of Egfr (Egfr-DN) similarly suppresses Ras ${ }^{12}$ tumour growth (e). Respective GFP (clones) channels are shown in the bottom panels (f-j). (k-n) Representative images showing terminal deoxynucleotidyl transferase (TdT) dUTP nick-end labelling (TUNEL) of Ras V12 or Ras V12, Egfr ${ }^{-}$double mutant clones to detect apoptotic cells. Ras ${ }^{V 12}$, Egfr ${ }^{-}$double mutant clones $(\mathbf{m}, \mathbf{n})$ do not show ectopic cell death compared to Ras ${ }^{V 12}$ clones (k,I). (o-r) Representative eye discs showing $\operatorname{Ras}^{V 12}$ (o) or Ras ${ }^{V 12}$, Egfr ${ }^{-}$double mutant (p) clones stained with anti-phosphohistone3 (PH3) antibodies to detect mitotic cells. Boxed areas are shown for comparison. Individual PH3 channels are shown in $\mathbf{q}, \mathbf{r}$. (s) Quantitation of $\mathbf{o}-\mathbf{r}$. The number of PH3-positive over total number of cells was scored in multiple clones across several animals for each genotype. The Egfr- mutation reduced the percentage of proliferative cells (Mean \pm s.d.\%, $N, P: 9.97 \pm 5.6 \%, N=1,693$ cells from 7 discs, $P<0.001$ versus $27.7 \pm 5.2 \%, N=2,100$ cells from 11 discs for Ras ${ }^{V 12}$ and Ras ${ }^{V 12}$, Egfr ${ }^{-}$double mutant cells, respectively). Error bars represent standard deviation (s.d.) from the mean for each genotype analysed. $P$ is derived from $t$-test analyses and $N$ denotes the sample size.

Egfr promotes $\operatorname{Ras}^{V 12}$-mediated tumour overgrowth via ARF6. In an effort to further elucidate how Egfr promotes oncogenic Rasdriven tumour overgrowth, we knocked down Egfr effectors (Drk and Arf6) by RNAi and asked whether this suppress oncogenic Rasmediated tumour overgrowth, and identified Arf6 as a mediator of Ras tumour overgrowth. Arf6 belongs to a family of highly conserved small Ras-related GTP-binding proteins and mediates Egfr signalling in mammals and flies ${ }^{15,37-39}$. Arf6 knockdown in otherwise wild-type clones showed no detectable growth defects (Figs $1 \mathrm{a}$ and $3 \mathrm{~b}$ ) but potently suppressed Ras ${ }^{V 12}$ tumour overgrowth (3a-c; quantified in $3 e$ ). In addition, Egfr directly recruits Arf6 GEFs to stimulate Arf6 (refs 37,38). We examined the effect of knocking the known Arf6 GEFs steppke and loner ${ }^{38,40}$. Knockdown of loner, but not steppke, suppressed $R a s^{V 12}$ tumour overgrowth (Fig. 3a,d-f, and Supplementary Fig. 1e,h), mimicking the effect of blocking the function of spi or Egfr or Arf6. In addition, we over-expressed Egfr in wing discs and asked whether knocking down Arf6 suppresses Egfr-mediated overgrowth effect and found that it does (Supplementary Fig. 2). Taken together, these data support the notion that Egfr acts through Arf6. 

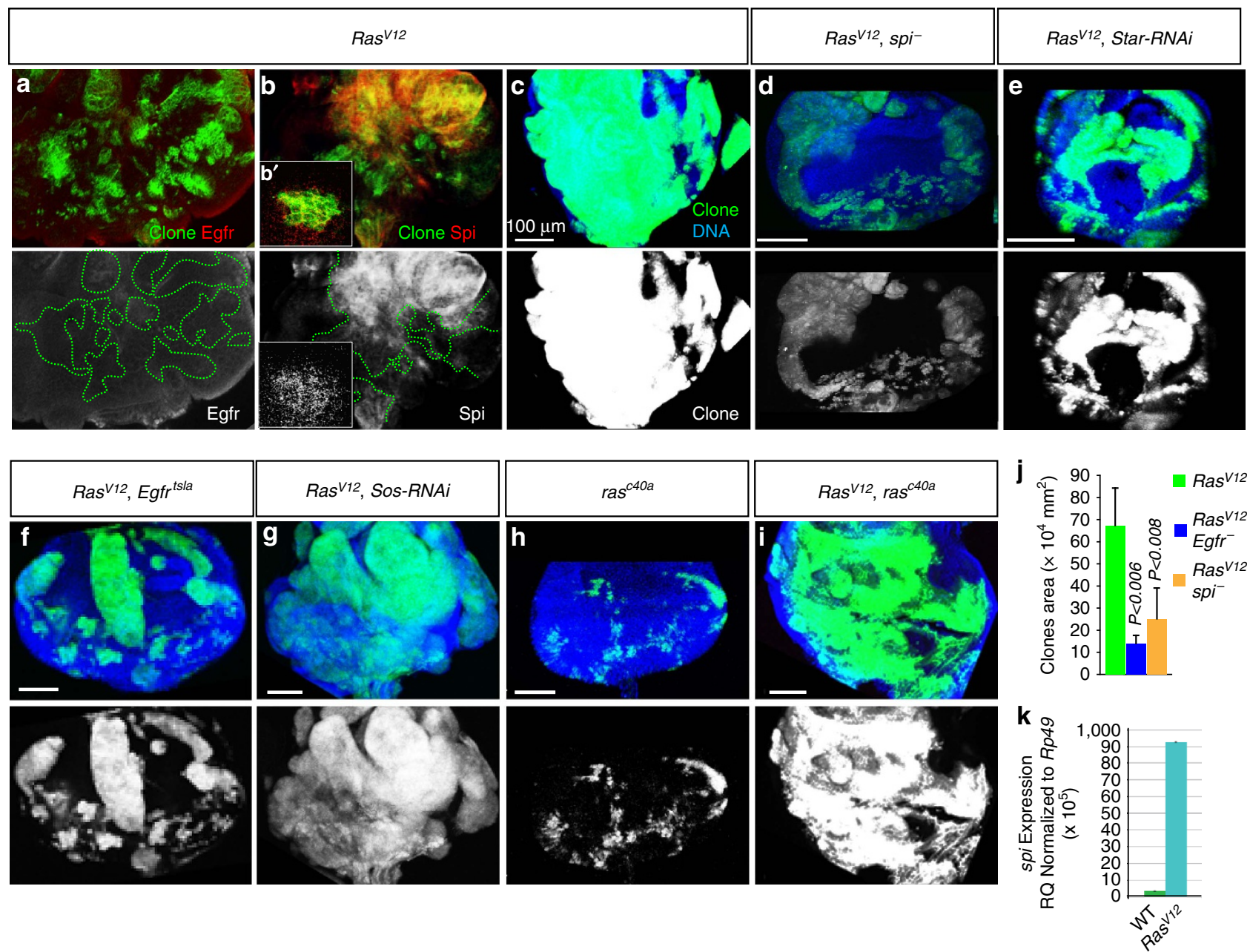

Figure 2 | Oncogenic Ras upregulates the expression of the EGFR ligand Spitz to stimulate tumour overgrowth independent of canonical EGFR signalling. (a-b') Early third-instar eye discs showing Ras ${ }^{V 12}$ clones (green) stained with anti-Egfr or anti-spitz antibodies (red). Egfr protein levels remain unchanged in Ras ${ }^{V 12}$ clones (a), while spitz is specifically elevated in Ras ${ }^{V 12}$ clones and can be detected around some clones (b, $\left.\mathbf{b}^{\prime}\right)$. (c $\left.\mathbf{c}-\mathbf{i}\right)$ Eye discs dissected from wondering third-instar animals showing clone growth for cells expressing Ras ${ }^{V 12}$ in otherwise wild-type background (c) or in cell carrying spi(d) or Egfr tsa (f) mutations or co-expressing Star-RNAi (e) or Sos-RNAi (g) or carrying the ras ${ }^{c 40 a}$ (i) mutation. $\mathbf{h}$ is showing the growth of ras ${ }^{c 40 a}$ clones in similarly aged animals. To the exception of Ras ${ }^{V 12}$, Egfr tlsa animals, which were raised at $29^{\circ} \mathrm{C}$, all animals were raised at $25^{\circ} \mathrm{C}$. spi ${ }^{-}$(d) Star-RNAi (e) and Egfr ${ }^{\text {tsa }}(\mathbf{f})$, but not Sos-RNAi (g), suppress the overgrowth of Ras ${ }^{V 12}$ clones (b). Similarly, ras ${ }^{\mathrm{c} 40 a}$ produced small clones (h) but fail to suppress the overgrowth of Ras ${ }^{V 12}$ tumour overgrowth (i). (j) Clone size quantitation. For this and all the subsequent clone size measurements, confocal stacks from similarly aged animal of the indicated genotypes and the image analysis software Imaris were used to measure clones areas. Mean \pm s.d.\%, $N, P: 13.8 \pm 3.5 \times 10^{4}, N=134$ clones, $P<0.006$ (Ras V12, Egfr ${ }^{-}$double mutant clones) or $24.9 \pm 13.8 \times 10^{4}, N=91$ clones $^{4} P<0.008$ (Ras ${ }^{V 12}$, spi ${ }^{-}$double mutant clones) versus $67.1 \pm 16.8 \times 10^{4}, N=20$ clones (Ras ${ }^{V 12}$ clones). (k) Reverse transcription PCR experiment measuring spi levels relative to expression levels of the housekeeping gene Rp49. Normalized fold differences of spi expression levels in eye discs bearing wild-type versus Ras ${ }^{12}$ clones. Experiments were performed in triplicates and standard deviations were derived from the coefficient variations of experimental and control samples. $P$ is derived from $t$-test analyses and $\mathrm{N}$ denotes the sample size.

Given our fly tumour data, we tested whether Arf6 serves as a molecular link in the cooperation between oncogenic Ras and Egfr in human cancer cells. We knocked down Arf6 in various lung and colon cancer cell lines with oncogenic Ras mutations. We found that Arf6-RNAi specifically reduced Arf6 protein levels and suppressed the growth of these cancer cells in comparison to cancer cells without oncogenic Ras mutations or cells treated with RNAi control (Fig. 3g,h, and Supplementary Fig. 3a). We conclude that in both Drosophila and human cells, Arf6 has a critical role in the growth of Ras mutant tumours.

Arf6 regulates Hh signalling to stimulate Ras tumour overgrowth. We sought to define the mechanism by which Arf6 drives the overgrowth of Ras ${ }^{V 12}$ tumours. Hh signalling is a good candidate because it cooperates with Egfr to drive basal cell carcinoma and melanomas via an unknown mechanism ${ }^{11,12}$. Consistent with the possibility that $\mathrm{Hh}$ signalling could be involved in Egfr/Arf6-mediated Ras tumour overgrowth, we found that Hh was upregulated and co-localized with Arf6 in Ras ${ }^{V 12}$ clones (Supplementary Fig. 4). We assessed the status of Hh signalling by examining the expression levels of its transcriptional target Cubitus interuptus (Ci or Gli in vertebrates) in complementing biochemical and immunostaining experiments. Using antibody against active $\mathrm{Ci}$, we found that $\mathrm{Ci}$ protein levels were elevated in lysates derived from dissected $\mathrm{Ras}^{V 12}$ discs compared with controls (Fig. 4a). Next, we directly stained wild-type or Ras ${ }^{V 12}$ mosaic tissues against $\mathrm{Ci}$. In wild-type discs $\mathrm{ci}$ is suppressed posteriorly while $\mathrm{Hh}$ activates ci anteriorly (Fig. 4g: bottom and top brackets, respectively) ${ }^{41}$. In contrast, 

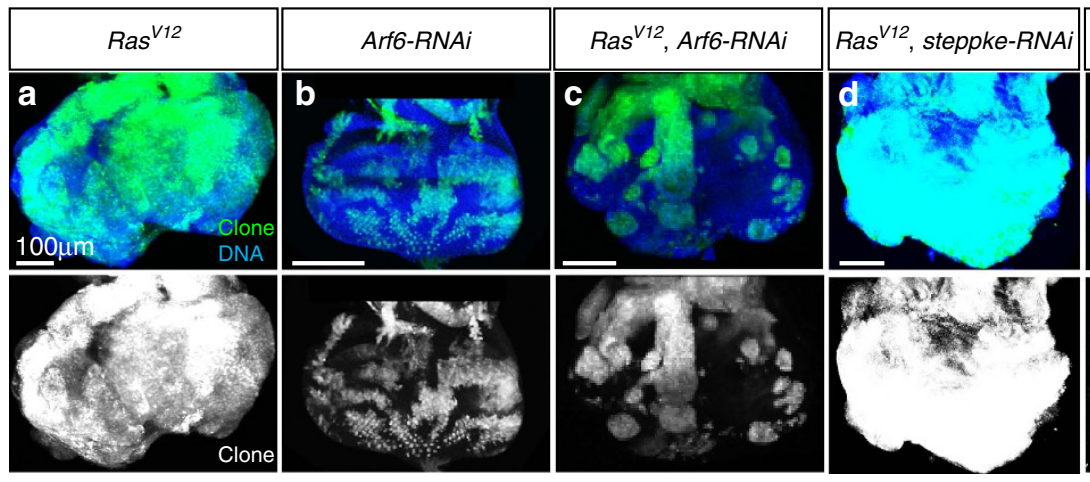

g

$(-)$ Scramble RNAi

(+) Arf6 RNAi

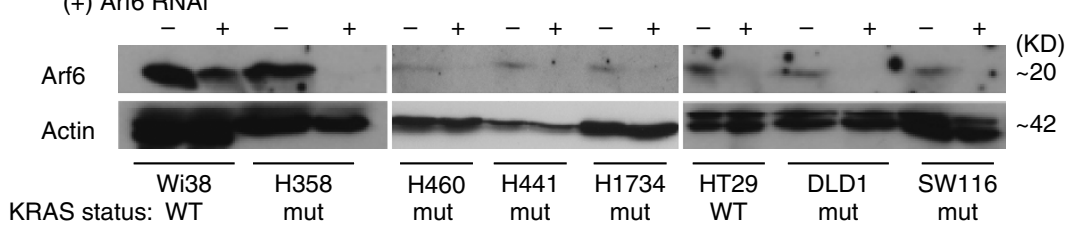

$\mathbf{f}_{\text {Ras }^{V 12}}$

Ras $^{\text {V12 }}$, Arf6-RNAi

Ras $^{\text {V12 }}$, loner-RNAi

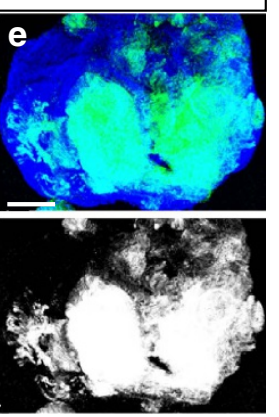

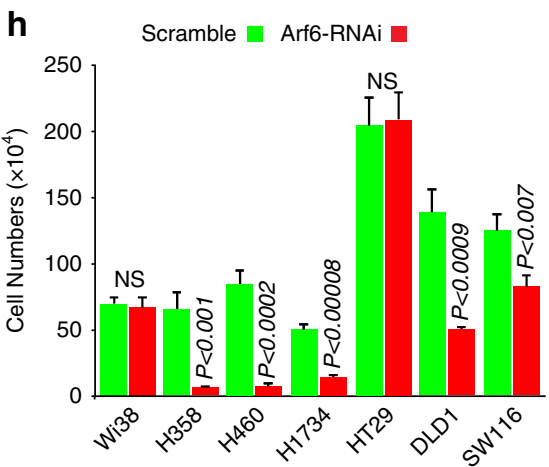

Figure 3 | Arf6 ${ }^{-}$suppresses oncogenic Ras tumours in flies and human cancer cells. (a-e) Representative images of wondering third-instar larval eye discs showing the growth of $\operatorname{Ras}^{V 12}$ (a) or Arf6-RNAi (b) clones or Ras ${ }^{V 12}$ clones co-expressing either Arf6-RNAi (c) or steppke-RNAi (d) or loner-RNAi (e). All animals were raised at $25^{\circ} \mathrm{C}$. Arf6-RNAi (b) causes no detectable growth defects but suppresses Ras ${ }^{V 12}$ tumour overgrowth (c). loner-RNAi (e), but not steppke-RNAi (d), blocks the overgrowth of Ras ${ }^{V 12}$ clones (d). (f) Quantitation of (a-d). Clones areas were $17.3 \pm 3.4 \times 10^{4}, N=128$ clones, $P<0.008\left(R a s^{V 12}\right.$ clones expressing Arf6-RNAi) or $31.7 \pm 8.1 \times 10^{4}, N=110, P<0.009$ (Ras ${ }^{V 12}$ clones expressing loner-RNAi) versus $67.1 \pm 16.8 \times 10^{4}, N=20$ clones $\left(R a s^{V 12}\right.$ clones). (g) Western blotting of various cancer cell lines treated with scramble or Arf6 RNAi and blotted with anti-Arf6 to detect Arf6 protein levels or anti-actin as a loading control. Cancer cells harbouring oncogenic Ras are denoted by mut while cells with wild-type Ras are indicated by WT. ARF6 protein levels are considerably reduced in cells treated with Arf6 RNAi. (h) Quantitation of cell numbers of lung and cancer cell lines 2 days after transfection with either control (scramble) or Arf6 RNAi. Not significant (NS) denotes $P$-value $>0.5$. $P$ is derived from $t$-test analyses and $N$ denotes the sample size.

ci was upregulated in posterior $\operatorname{Ras}^{\mathrm{V} 12}$ clones and this upregulation was more pronounced in larger clones (see discussion) (Fig. 4b,c, and corresponding bottom panels). In addition, expression of $\operatorname{Ras}^{V 12}$ in wing discs, showed ectopic ci activation (Supplementary Fig. 5). We conclude that oncogenic Ras activates Hh signalling.

The overexpression of ci in eye disc clones correlated with increased cell proliferation as determined by phospho-histone 3 immunostaining (Fig. 4b and bottom panel). Knockdown of Arf6 suppressed $\mathrm{Hh}$ signalling in similar size clones and correspondingly reduced cell proliferation in $\operatorname{Ras}^{V 12}$ clones (Fig. 4b,c and t). Removing Spi or Egfr function in $\operatorname{Ras}^{V 12}$ clones (Ras ${ }^{V 12}$, spi- or Ras $^{V 12}$, Egfr- double mutant cells) showed similar effects (Fig. 4b,c,e, and f), consistent with the notion that Egfr/Arf6 signalling stimulates $\mathrm{Hh}$ signalling in Ras tumours.

We have previously shown that tumours co-opt developmental mechanisms to promote overgrowth ${ }^{25}$. During development, dying cells upregulate Jun N-terminal kinase (JNK) signalling, which in turns instructs neighbouring cells to activate Janus kinase/signal transducers and activators of transcription (JAK-STAT) signalling and proliferate in order to maintain tissue homeostasis. Ras ${ }^{V 12}$ cells usurp this compensatory cell proliferation mechanism by forcing JNK activation in surrounding wild-type cells via elevated secretion of JNK signalling ligands, leading to tumour overgrowth ${ }^{25}$. We thus examined whether Arf6 regulates $\mathrm{Hh}$ signalling during normal development. In the developing eye imaginal disc, anterior cells activate $\mathrm{Hh}$ signalling in response to $\mathrm{Hh}$ secreted from the posterior cells (Fig. $4 \mathrm{~h}$ and ref. 41). Similarly, the engrailed gene is specifically expressed in the posterior compartment $(\mathrm{P})$ of the developing wing disc where it induces $\mathrm{Hh}$ expression but represses $\mathrm{ci}^{42}$. Posterior cells relay $\mathrm{Hh}$ to the anterior compartment (A) where $\mathrm{Hh}$ activates target genes including $c i$ and patched (ptc) to control wing size and patterning ${ }^{43,44}$. We generated Arf6-RNAi clones in developing eye and wing discs, examined Hh signalling in the Hh responsive anterior cells, and found that Arf6-RNAi suppressed Hh signalling in both tissue types (Fig. $4 \mathrm{~g}$ and bottom panel, bracket, and j; respectively). Conversely, we overexpressed Arf6 in wing discs using the dorsal driver apterus-GAL4 (ap-GAL4) and asked whether this upregulates $\mathrm{Hh}$. Posteriorly produced Hh normally activates ptc in a 8-10 cells diameter domain along the $\mathrm{A} / \mathrm{P}$ boundary. Consistent with enhanced $\mathrm{Hh}$ signalling, ap-Gal4 >UAS-ARF6 discs showed elevated Ptc protein levels and an expansion of the Ptc domain specifically in the apterusdorsal portion of the disc (Fig. 4k).

Next, we directly knocked down Arf6 along the A/P compartments boundary where high levels of $\mathrm{Hh}$ signalling regulate the patterning and growth of the wing. Arf6 knockdown interfered with the formation of the anterior cross vein and that of the longitudinal vein 3 (L3), a processes regulated by Hh signalling ${ }^{45}$ (Fig. 4l,m, and arrowhead). Importantly, Arf6 knockdown 

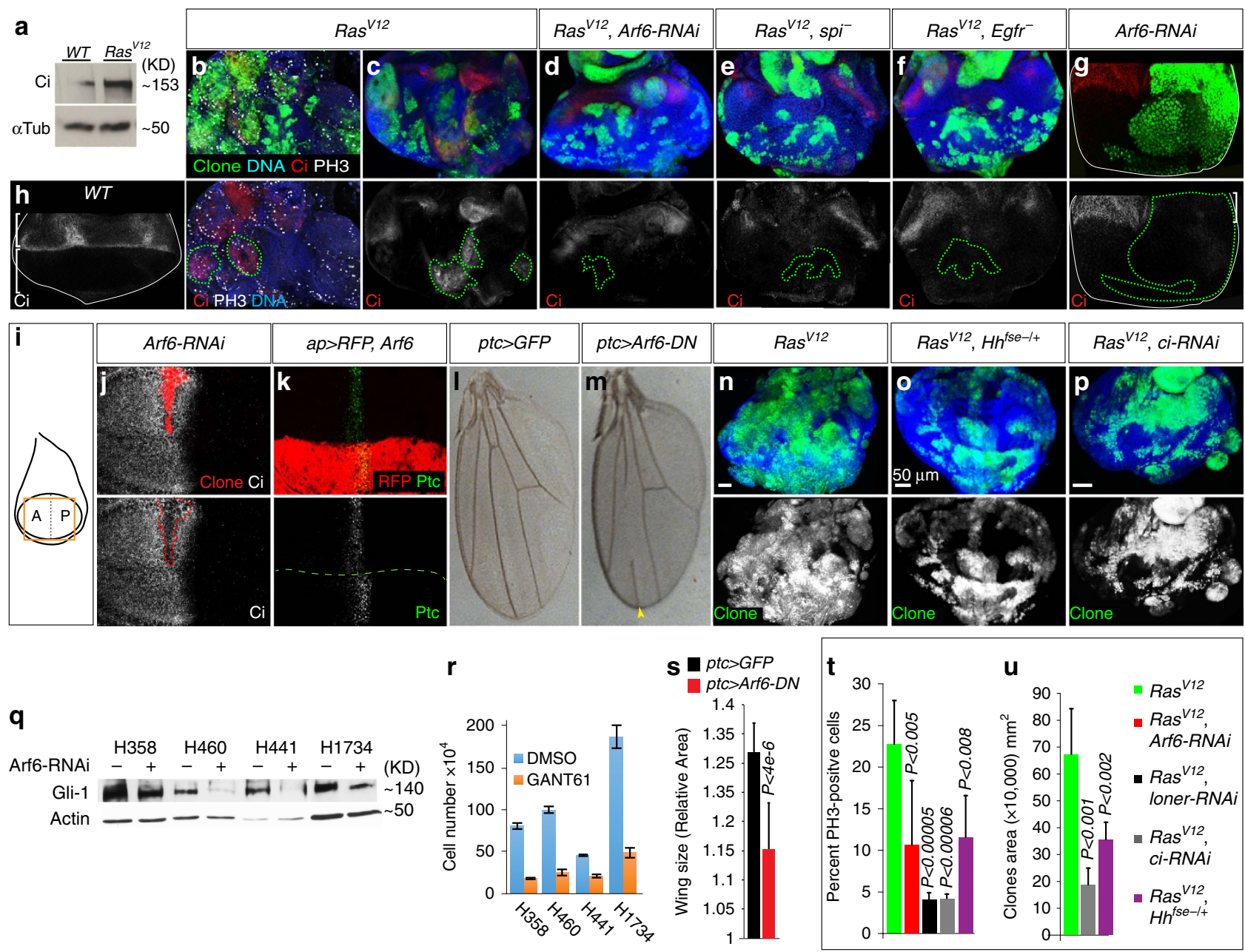

Figure 4 | Arf6 regulates Hedgehog signalling to control tumour overgrowth. a) Protein lysates derived from dissected wild-type discs or discs with RasV12 clones were used in western-blotting experiments to detect active (full-length) Ci protein levels against the loading control ( $\alpha$-tubulin). (b-f) Early 3rd-instar eye discs showing ey-FLP MARCM clones of the indicated genotype stained with DAPI, anti-Ci, and/or anti-PH3. Bottom panels show PH3 and/ or Ci separately. In this and all the remaining panels, dotted lines denotes clones boundary. (g) Early 3rd-instar eye disc showing hs-FLP MARCM Arf6-RNAi clones stained with anti-Ci. The bottom panel shows the $\mathrm{Ci}$ channel. h) Wild-type eye disc stained against $\mathrm{Ci}$. The top bracket denotes the zone of high $\mathrm{Hh}$ signalling in the eye. (i) Schematic of a wing disc. The box illustrates the region of the disc shown in ( $\mathbf{j}$ and $\mathbf{k}$ ). $\mathbf{j}$ ) Early $3 r d$-instar wing disc showing hs-FLP Arf6-RNAi MARCM clones (red) and stained against $\mathrm{C}$. The $\mathrm{Ci}$ channel is shown in the bottom panel. (k) Expression of Arf6-RNAi in the dorsal domain (RFP) of the wing pouch in early 3rd-instar animals using apterus-GAL4 and stained with anti-patched. Bottom panel shows patched channel alone. The dotted line delimits the dorsal-apterus domain in the wing pouch. (I and $\mathbf{m}$ ) Ptc-GAL4 expression of GFP or Arf6-DN (n-p) Similarly-aged eye discs showing clone growth for cells expressing Ras ${ }^{V 12}$ alone (n) or with $\mathrm{Hhfse}^{-/+}(\mathbf{o})$ or ci-RNAi (p). Clone channels are shown in bottom panels. (q) Western blotting of various lung cancer cell lines treated with scramble or Arf6 RNAi and blotted with anti-Gli1 or anti-actin. (r) Lung cancer cell lines treated either with DMSO or GANT61. (s) Quantitation of relative wing sizes from $\mathbf{o}$ and $\mathbf{p}$. (t) Quantitation of the percentage of PH3-positive cells in clones of the indicated

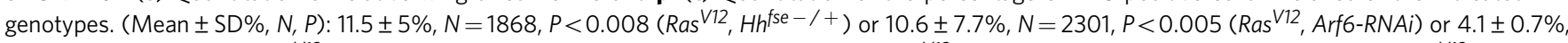
$N=1764, P<0.00005$ (Ras V12, loner-RNAi) or $4.15 \pm 0.5 \%, N=6738, P<0.00006$ (Ras V12, ci-RNAi) versus $27.7 \pm 5.2 \%, N=2100$ for Ras ${ }^{12}$. u) Quantitation of (n-p). (Mean \pm SD\%, N, P): $35.3 \pm 6.3 \times 10^{4} \mathrm{~mm}^{2}, N=171$ clones, $P<0.002$ (Ras ${ }^{V 12}, \mathrm{Hh}^{f s e-/+}$ ) or $18.5 \pm 6.1 \times 10^{4} \mathrm{~mm}{ }^{2}, \mathrm{~N}=58 \mathrm{clones,}$ $P<0.001$ (Ras ${ }^{V 12}$, ci-RNAi). $P$ is derived from $t$-test analyses and $N$ denotes the sample size.

significantly reduced wing size compared with controls $($ ptc-GAL4 $>$ GFP) (Fig. 4l,m,s, and Supplementary Fig. 6). Taken together, these data indicate that Arf6 regulates Hh signalling in Ras tumours and during development.

We subsequently tested whether Egfr/Arf6-triggered Hh signalling synergizes with oncogenic Ras to cause tumour overgrowth. We analysed the growth of $\mathrm{Ras}^{\mathrm{V} 12}$ clones induced in tissues heterozygotes for a hypomorphic allele of $\mathrm{Hh}, \mathrm{Hh}^{\text {fse }}$ (ref. 46), thus reducing Hh function by less than 50\% compared with controls. We found that this considerably blocked cell proliferation and the overgrowth phenotype of Ras ${ }^{V 12}$ clones (Fig. 4n,o; quantified in $\mathrm{t}$ and $\mathrm{u}$ ). RNAi knockdown of ci showed a similar effect (Fig. 4n,p; quantified in $t$ and $u$ ). Egfr/Arf6triggered $\mathrm{Hh}$ signalling thus synergizes with oncogenic Ras to cause tumour overgrowth.

Finally, we determined whether Arf6 similarly regulates Hh signalling in human cancer lines by examining changes in Gli1 expression levels following Arf6 RNAi knockdown. We focused on lung cancer cell lines because Arf6 knockdown potently inhibited growth in these cancer cells. Arf6 RNAi efficiently knocked down Arf6 protein levels (Fig. 3g) and reduced Gli1 levels in lung cancer cells (Fig. $4 \mathrm{q}$ and Supplementary Fig. 9). Direct inhibition of Hh signalling using GANT61, a specific Gli1 small molecule inhibitor ${ }^{47,48}$, suppressed 
growth similar to Arf6 knockdown (Fig. 4r). Taken together, we conclude that Arf6 regulates Hh signalling in both flies and human cancer cells to control growth.

Arf6 controls Hh trafficking. We investigated the relationship between EGFR, ARF6 and Hh using immunostaining and immunoprecipitation experiments. We previously observed that Arf6 co-localizes with Hh in Ras ${ }^{V 12}$ cells (Supplementary Fig. 4) and thus wondered whether Egfr modulates Ras ${ }^{V 12}$ tumour overgrowth by regulating an interaction between Arf6 and Hh. We performed Arf6 pull-down experiments in the presence or absence of Egfr and found that abrogating Egfr function in Ras ${ }^{V 12}$ cells diminishes Arf6's ability to pull-down Hh (Fig. 5a and Supplementary Fig. 7a), indicating that Egfr promotes the interaction of Arf6 with Hh. We then explored a mechanism for Arf6 regulation of $\mathrm{Hh}$. Endocytosis and vesicle transport are known to control Hh signalling ${ }^{13}$. We hypothesized that Arf6 could promote the shuttling of $\mathrm{Hh}$ to signalling competent endosomes. In the absence of Arf6, Hh would be routed to the degradation pathway hence causing suppression of Hh signalling. The Hepatocyte growth factor-regulated tyrosine kinase substrate (Hrs) binds to and directs signalling molecules to the lysosomal degradation pathway to downregulate signalling ${ }^{49}$. We examined Hh localization to Hrs-positive vesicles and found that Arf6 knockdown caused Hh to predominantly localize to Hrs-positive vesicles compared with controls (Fig. 5b,e and f). Knocking down Egfr in Ras ${ }^{V 12}$ cells showed similar effects (Supplementary Fig. 7b-d). In addition, we directly used late endosomal markers (Arl8 and Rab7) to test whether Arf6 knockdown causes trafficking of Hh to lysosomal vesicles. We could not co-stain tissues with anti-Hh and anti-Arl8 (or anti-Rab7) antibodies because these antibodies were raised in the same species. Instead we used the GMR-GAL4 driver to express GFP-labelled full-length or a version of Hh corresponding to its secreted and active form (Hh-GFP and Hh-N-GFP, respectively) in cells expressing either Ras ${ }^{V 12}$ alone or co-expressing Arf6-DN or Arf6-RNAi, and stained tissues against Arl8. We found that Hh-GFP preferentially localizes to clusters of Arl8-positive vesicles in Ras ${ }^{V 12}$, Arf6-DN tissues (Fig. 5c,c' and g). Similarly, we expressed Rab7-GFP in Ras ${ }^{V 12}$, Arf6-DN cells, stained against $\mathrm{Hh}$, and found that Hh predominantly localizes to Rab7-GFP vesicles in these cells compared with controls (Ras ${ }^{V 12}$ cells) (Fig. 5d,h versus i,m). Moreover, expression of Arf6-RNAi in Ras ${ }^{V 12}$ cells resulted in the redistribution of Hh-N-GFP to Arl8 endosomes (Fig. 5j,n versus k,o). Furthermore, expression of Arf6-RNAi in otherwise wild-type cells similarly showed Hh localization to Rab7 endosomes (Supplementary Fig. 7e,f and h). Consistent with this, Patched (Hh receptor) localizes to Rab7 or Arl8 endosomes in Arf6-RNAi or Arf6-DN expressing cells (Fig. 5l,p, and Supplementary Fig. 6g-j). Together with the Hh-Hrs colocalization results above, these data indicate that Arf6 normally prevents the trafficking of $\mathrm{Hh}$ to the degradation pathway. Thus, Arf6 controls Hh trafficking in order to promote Hh signalling, which in turn cooperates with Ras ${ }^{V 12}$ to synergistically stimulate tumour overgrowth.

\section{Discussion}

How oncogenic Ras signalling elicits Egfr and Hh functions and how these distinct signalling events are integrated to achieve oncogenic synergy in cancers is not well understood. Using a Drosophila tumour model we found that oncogenic Ras transcriptionally stimulates the TGF- $\alpha$ Egfr ligand spitz to recruit Egfr signalling and increase tumour growth. Egfr's role is likely tissue and/or context-dependent as inhibition of Egfr shows variable effects in different cancer types ${ }^{4,5,50}$. Interestingly,
Egfr inhibition in Ras ${ }^{V 12}$ appeared to cause a non-autonomous growth effect (Fig. 1a,d and e), suggesting that JNK signalling is involved $^{51}$. However, blocking JNK signalling fails to rescue the growth of $\mathrm{Ras}^{\mathrm{V} 12}$, Egfr ${ }^{-}$clones (Supplementary Fig. 8). Thus Egfr ${ }^{-}$suppresses Ras ${ }^{V 12}$ tumour overgrowth independent of JNK signalling. Instead, further analyses unexpectedly uncovered a non-canonical signalling modality for Egfr in $\operatorname{Ras}^{V 12}$ cells. Rather than signalling via the MAPK pathway, Egfr acts through Arf6. Knockdown of Arf6 in flies or human cancer cells suppresses the overgrowth of cell harbouring oncogenic Ras. This effect is due to a cell proliferation defect because, similar to Egfr knockdown, Arf6 knockdown showed no ectopic cell death in cancer lines with the highest growth suppression effect (Supplementary Fig. 2b).

We show that Arf6 acts via Hh signalling. Egfr promotes Arf6 to interact with $\mathrm{Hh}$ in order to stimulate $\mathrm{Hh}$ signalling. Arf6 stimulates $\mathrm{Hh}$ signalling by protecting $\mathrm{Hh}$ from lysosomal degradation. Consistent with this, Arf6 regulates the formation of carrier vesicles and interacts with lysosomes to control vesicle trafficking in epithelial cells ${ }^{52,53}$. We show that disrupting the interaction between Arf6 and Hh by either blocking Egfr or by directly depleting Arf6 causes Hh to accumulate in lysosomal vesicles. Interestingly, Arf6-depleted cells also show an increased number of Hrs-positive endosome overall, suggesting that Arf6 may impact other unknown signalling molecules in addition to $\mathrm{Hh}$.

Hh signalling is activated in Ras tumour cells, and it is especially higher in larger clones. This could be because bigger clones produce more Spi and $\mathrm{Hh}$ in the clones' milieu allowing Arf6 to drive $\mathrm{Hh}$ signalling robustly in these cells. Importantly, blocking Egfr or Arf6 suppresses Hh signalling and correspondingly inhibits overgrowth in both flies and human cancer cells. Direct inhibition of Hh signalling suppresses the growth of fly and human cancer cells. Indeed we found that the overgrowth of Ras tumours is hypersensitive to Hh signalling dosage. Partial reduction of $\mathrm{Hh}$ protein is sufficient to effectively block tumour overgrowth, consistent with a synergetic cooperation. Thus Egfr/Arf6 has an important role in bridging oncogenic Ras and Hh signalling pathways.

Finally, we found that Arf6 regulates Hh signalling during normal development. ARF6 knockdown inhibited Hh signalling in developing imaginal discs and impinged on Hh signallingregulated developmental processes.

Together our data highlight a non-canonical Egfr signalling mechanism centered on a novel role for Arf6 in Hh signalling regulation. We define a novel signalling mechanism that explains the perplexing requirement of Egfr in oncogenic Ras-mediated overgrowth and the oncogenic cooperation between Egfr and Hh signalling.

\section{Methods}

Experimental procedures. Fly lines. Animals were aged at $25^{\circ} \mathrm{C}$ on standard medium. The following fly lines were used in this study: (1) $y w$; FRT82B; (2) $y w$; FRT82B, UAS-Ras ${ }^{V 12} / T M 6 B$; (3) EgfrTop-CA (T. Schupback, Princeton Univ.); (4) UAS-DER(DN) (A. Michelson, Harvard Univ.); (5) UAS-spi-RNAi (VDRC \# 3922); (6) cn1 Egfr ${ }^{\text {tsla }}$ bw1/T(2;3)TSTL, CyO: TM6B, Tb1(BDSC\# 6501);(7) FRT40, ras ${ }^{40 A}$ (C. Berg, Univ. of Washington); UAS-ARF6-RNAi (VDRC \# 24224, 100726; BDSC\# 51417, 27261); (8) UAS-loner-RNAi (VDRC \#106168 and BDSC\#39060); (9) UAS-ci-RNAi (BDSC\# 28984); (10) spi, FRT40A/CyO (BDSC\#114338); (11) yw,eyFlp1;act $>y+>$ GAL4,UAS-GFP.S65T;FRT82B,tub-GAL80; (12) $\mathrm{Hh}^{\text {fse }}$ (BDSC\# 35562); (13)yw,ey-Flp1;act >y+>GAL4,UAS-myrRFP;FRT82B,tub-GAL80; (14) yw,ey-Flp1; FRT42D,tub-GAL80; act >y+ > GAL4,UAS-GFP; (15) UAS-ARF6DN (E. Chen, Johns Hopkins Univ.); (16)yw,ey-Flp1;tub-GAL80,FRT40A;act $>y+>$ GAL4,UAS-GFP.S65T; (17) Star-RNAi (BDSC\# 38914); (18) Sos-RNAi (BDSC\#31174); (19) Arf6-GFP line is a previously generated and functionally validated GFP knock-in line $\mathrm{e}^{54,55}$ kindly provided by Y. Hong (University of Pittsburg). In brief, the Arf6 gene is under endogenous control and a GFP is inserted in frame at the carboxyl terminus of Arf6 protein; (20) UAS-steppke-RNAi (BDSC\#32374); (21) UAS-Hh-GFP and UAS-Hh-N-GFP, a gift from A. O'Reilly (Fox Chase Cancer Center, PA); (22) yw, hsp70-Flp; act- $y^{+}$-GAL4, UAS-GFP. See Supplementary Note 1 for genotype details of all images. 
a
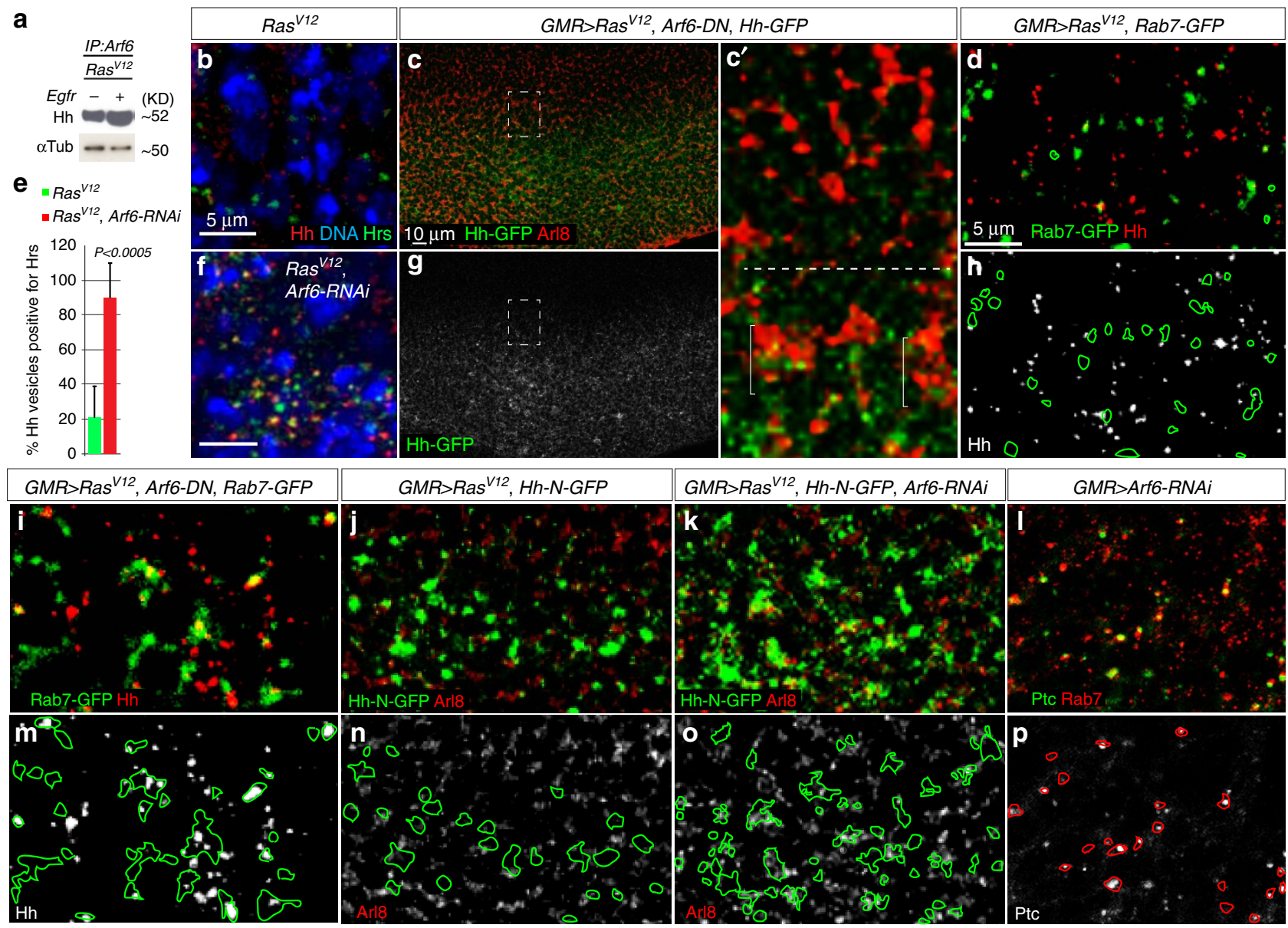

Figure 5 | Arf6 controls Hedgehog cellular trafficking. (a) Protein lysates derived from dissected wild-type discs or discs with Ras 712 tumours were used in western-blotting experiments to detect full-length (active) Cubitus interuptus (Ci) protein levels against the loading control ( $\alpha$-tubulin). Hh co-precipitates with Arf6 but the Egfr ${ }^{-}$mutation interferes with Arf6's ability to interact with Hh. (b,f) Ras ${ }^{\mathrm{V} 12}$ or Ras ${ }^{\mathrm{V} 12}$, Arf6-RNAi co-expressing cells stained with anti-hedgehog, anti-Hrs antibodies and DAPI (4,6-diamidino-2-phenylindole). Distinct Hh (red) or Hrs (green)-positive vesicles are detected in Ras ${ }^{V 12}$ cells (b). In contrast, Hh predominantly localizes to Hrs-positive vesicles in Ras V12, ARF6-RNAi co-expressing cells (f). (c) Eye disc co-expressing Hh-GFP, Ras V12 and Arf6-DN under the control of GMR-GAL4 and stained against Arl8. The Hh-GFP channel alone is shown in $\mathbf{g}$. Higher magnification image of posterior/anterior boundary (boxed area) is shown in c'. Hh-GFP localizes to clusters of Arl8-positive vesicles specifically in the posterior portion of the eye disc (brackets), but not in the anterior region of the eye (above the dotted line). (d,h,l,m) Images from eye disc co-expressing either Ras 172 and Rab7-GFP (d,h) or Ras V12, Arf6-DN, and Rab7-GFP (i,m) using GMR-GAL4 and stained with anti-Hh antibodies. Images of the Hh channel for $\mathbf{d}, \mathbf{i}$ are shown in $\mathbf{h}, \mathbf{m}$, respectively. Fluorescent signal contours of Rab7-GFP $(\mathbf{m})$ and Hh-N-GFP $(\mathbf{n}, \mathbf{o})$ are shown in $\mathbf{m}-\mathbf{0}$. (e) Quantitation of Hh punctae localizing to Hrs-positive vesicles. The total number of $\mathrm{Hh}$ punctae in Ras ${ }^{V 12}$ or Ras ${ }^{V 12}$, Arf6-RNAi co-expressing cells ( $N=27$ and 32, respectively) was scored for each genotype and the respective percentage of $\mathrm{Hh}$ localizing to Hrs-positive vesicles was determined. (j,k) Images from an eye disc co-expressing Ras ${ }^{V 12}$ and the secreted form of $\mathrm{Hh}$

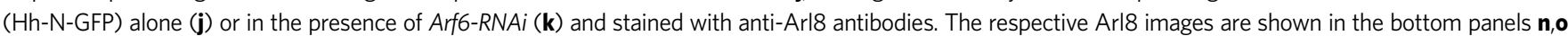
(I) Image from an eye disc expressing Arf6-RNAi with GMR-GAL4 and stained against patched (ptc) and Rab7. The corresponding patched channel is shown alone in $\mathbf{p}$. Rab7 fluorescent signal contours are shown in $\mathbf{p}$. $P$ is derived from t-test analyses and $N$ denotes the sample size.

Clones. Clones were generated using standard MARCM techniques ${ }^{56}$ Detailed genotypes for all images are included in Supplementary Information. Briefly, all clones were induced with $e y-F L P$ MARCM system, to the exception of Fig. 4g,j, which were generated with $h s$-Flp. For these particular experiments, 2 days larvae were heat-shocked for $1 \mathrm{~h}$ at $37^{\circ} \mathrm{C}$ and raised for two days at $25^{\circ} \mathrm{C}$ before processing. Images presented in Figs $2 \mathrm{f}, 41, \mathrm{~m}$, and Supplementary Figs $1 \mathrm{k}, \mathrm{n}, 2,5$ and 6 were obtained from animal raised at $29^{\circ} \mathrm{C}$. For all remaining images, animals were raised at $25^{\circ} \mathrm{C}$. Clone size was obtained by determining the average clone area in discs dissected from randomly selected wondering third-instar control versus experimental larvae using the image analysis IMARIS Properly aged GFP-positive mosaic animals were randomly and blindly selected under a dissecting microscope using white light to eliminate bias. Minimum sample sizes were determined using a $5 \%$ confidence level and $80 \%$ power. The minimum sample sizes were then used to estimate the number of animals/discs to be examined.

Staining and imaging. Eye-antenna discs were dissected, fixed and stained as described previously ${ }^{25,26}$. Tumour and adult eyes sizes analyses were carried out on a Leica MZ FLIII fluorescence stereomicroscope equipped with a camera.
Samples were examined by confocal microscopy with a Zeiss LSM510 Meta system and a Leica TCS SP8 confocal microscope. Images were analysed and processed with IMARIS (Bitplane, Switzerland) and Illustrator (Adobe) software, respectively. The following primary antibodies were used: guinea pig anti-Hrs (1:2,000, H. Bellen); Mouse anti-Spi (1:500; A.D. Vrailas-Mortimer); Rabbit anti-phosphohistone-H3 (1:1,000, Sigma); Rat anti-DER (1:1,000, B. Shilo, Weizmann Institute of Science/Israel); Mouse anti-EGFR (1:500, abcam); Rabbit anti-GFP (1:1,000, Abcam); Rabbit anti-Hh (1:500 Pre-absorbed, T. Kornberg, UCSF); Rat anti-Ci (detects the full length/active version of $\mathrm{Ci}$, 1:200, Developmental Studies Hybridoma Bank, 2A1); Mouse anti-Patched (1:200, Developmental Studies Hybridoma Bank, Apa1). Secondary antibodies were from Invitrogen. TUNEL staining was performed using the Apoptag Red kit from Chemicon.

Western blots and immunoprecipitation. For immunoprecipitation experiments, imaginal discs from 50 third-instar animals carrying Ras ${ }^{V 12}$ single or Egfr ${ }^{T o p}-C A$ RasV12 or DerDN(EGFR-DN), Ras ${ }^{V 12}$ double mutant clones were homogenized in lysis buffer (50 mM HEPES (pH 7.5), $150 \mathrm{mM} \mathrm{KCl,} 5 \mathrm{mM} \mathrm{MgCl}, 0.01 \%$ Triton-X) 
supplemented with protease inhibitor tablets; Roche). Global protein concentrations was normalized across all genotypes and the lysate was pre-cleared with protein agarose-A beads for $1 \mathrm{~h}$ at $4^{\circ} \mathrm{C}$, incubated either with $2 \mu \mathrm{l}$ of anti-Arf6 (M. Gonzalez-Gaitan) for $4 \mathrm{~h}$ at $4{ }^{\circ} \mathrm{C}$. Lysates were then incubated with protein agarose $\mathrm{A}$ beads for $1 \mathrm{~h}$ at room temperature. For pull downs, beads were precipitated and washed three times in modified lysis buffer containing 0.5\% TritonX-100. Samples were run on SDS-polyacrylamide gel electrophoresis, transferred on nitrocellulose membrane, and blotted with anti-Hh (T. Kornberg, UCSF) and anti- $\alpha$ Tubulin (Sigma), loading control. For cancer cell lines, cells were lysed in lysis-loading buffer (0.5 M Tris (pH 6.8), 20\% SDS, Glycerol, $10 \% \beta$-mercaptoethanol and Bromophenol Blue), homogenized further by hydrodynamic shearing (15 times through a 25 -gauge syringe, and centrifuged at 10,000 r.p.m. for $5 \mathrm{~min}$. The supernatant was collected and run on SDS-polyacrylamide gel electrophoresis, transferred on nitrocellulose membrane, and blotted with anti-Gli1 (1/1,000; Abcam, cat\# ab134906) or anti-Arf6 $(1 / 1,000$; Abcam, Cat\# ab77581) or anti $\beta$-actin (1/1,000; Sigma, cat\# A5441) antibodies. Full images of the blots are presented in Supplementary Fig. 9.

Real-time polymerase chain reaction. Total RNA from Eye-antenna imaginal discs containing wild-type or mutant clones was isolated using a Trizol RNA extraction method (Invitrogen). The SuperScriptIII First-Strand Synthesis System (Invitrogen) kit was used to synthesize complementary DNA. Real-time PCR was carried out on an Applied Biosystems machine using the SYBR green fast kit (Applied Biosystems) following the manufacturer's instructions. Relative gene expression was obtained from triplicate runs normalized to Rp49 as endogenous control. The following primers were used: $R p 49,5^{\prime}$-GGCCCAAG ATCGTGAAGAAG- $3^{\prime}$ and $5^{\prime}$-ATTTGTGCGACAGCTTAGCATATC-3'; Spitz, $5^{\prime}$-CGCCCAAGAATGAAAGAGAG- $3^{\prime}$ and $5^{\prime}$-AGGTATGCTGCTGGTG GAAC- $3^{\prime}$

Clones of mutant cells in the eye-antennal discs were generated using standard MARCM system ${ }^{56}$. Immunostaining and Immunoprecipitation experiments were performed as previously described ${ }^{57}$

ARF6 RNAi gene knockdown in human cancer cell lines. In all, $2 \times 10^{5}$ cells were seeded onto 6-well-plates and treated with $2 \mathrm{nM}$ of either ARF6 SiRNA (combination of three SiRNA of ARF6, Origene; Cat\# SR300275) or universal control scrambled (Origene, SiRNA Cat\# SR30004) and supplemented with $5 \mu \mathrm{l}$ of DhamaFect 2 (GE- Dharmacon).

Treatment of lung cancer cells with GANT61. Cells were seeded onto 6-well-plates at a density of $2 \times 10^{5}$ and grown overnight before GANT61 (Cat\# G9048-5MG, Sigma-Aldrich) treatment at the final concentration of $10 \mu \mathrm{m} \mathrm{ml}^{-1}$. Cell numbers were scored $96 \mathrm{~h}$ after GANT61 treatment.

Data availability. All data supporting the findings of this study are available within the article and its Supplementary Information files or from the corresponding author upon reasonable request.

\section{References}

1. Bos, J. L. ras oncogenes in human cancer: a review. Cancer Res. 49, 4682-4689 (1989).

2. Downward, J. Targeting RAS signalling pathways in cancer therapy. Nat. Rev. Cancer 3, 11-22 (2003).

3. Bardeesy, N. et al. Role of epidermal growth factor receptor signaling in RAS-driven melanoma. Mol. Cell. Biol. 25, 4176-4188 (2005).

4. Ardito, C. M. et al. EGF receptor is required for KRAS-induced pancreatic tumorigenesis. Cancer Cell 22, 304-317 (2012).

5. Navas, C. et al. EGF receptor signaling is essential for k-ras oncogene-driven pancreatic ductal adenocarcinoma. Cancer Cell 22, 318-330 (2012).

6. Liou, G. Y. et al. Mutant KRas-induced mitochondrial oxidative stress in acinar cells upregulates EGFR signaling to drive formation of pancreatic precancerous lesions. Cell Rep. 14, 2325-2336 (2016).

7. Casci, T. \& Freeman, M. Control of EGF receptor signalling: lessons from fruitflies. Cancer Metastasis Rev. 18, 181-201 (1999).

8. McCormick, F. Signal transduction. How receptors turn Ras on. Nature 363, 15-16 (1993).

9. Mimeault, M. \& Batra, S. K. Frequent deregulations in the hedgehog signaling network and cross-talks with the epidermal growth factor receptor pathway involved in cancer progression and targeted therapies. Pharmacol. Rev. 62, 497-524 (2010).

10. Stecca, B. et al. Melanomas require HEDGEHOG-GLI signaling regulated by interactions between GLI1 and the RAS-MEK/AKT pathways. Proc. Natl Acad. Sci. USA 104, 5895-5900 (2007).

11. Eberl, M. et al. Hedgehog-EGFR cooperation response genes determine the oncogenic phenotype of basal cell carcinoma and tumour-initiating pancreatic cancer cells. EMBO Mol. Med. 4, 218-233 (2012).
12. Ji, Z., Mei, F. C., Xie, J. \& Cheng, X. Oncogenic KRAS activates hedgehog signaling pathway in pancreatic cancer cells. J. Biol. Chem. 282, 14048-14055 (2007).

13. Gallet, A. \& Therond, P. P. Temporal modulation of the Hedgehog morphogen gradient by a patched-dependent targeting to lysosomal compartment. Dev. Biol. 277, 51-62 (2005).

14. Vetter, I. R. \& Wittinghofer, A. The guanine nucleotide-binding switch in three dimensions. Science 294, 1299-1304 (2001).

15. Donaldson, J. G. Multiple roles for Arf6: sorting, structuring, and signaling at the plasma membrane. J. Biol. Chem. 278, 41573-41576 (2003).

16. Bozza, G. et al. Role of ARF6, Rab11 and external Hsp90 in the trafficking and recycling of recombinant-soluble Neisseria meningitidis adhesin $\mathrm{A}(\mathrm{rNadA})$ in human epithelial cells. PLoS ONE 9, el10047 (2014).

17. D'Souza-Schorey, C., Li, G., Colombo, M. I. \& Stahl, P. D. A regulatory role for ARF6 in receptor-mediated endocytosis. Science 267, 1175-1178 (1995).

18. Lawrence, J. T. \& Birnbaum, M. J. ADP-ribosylation factor 6 delineates separate pathways used by endothelin 1 and insulin for stimulating glucose uptake in 3T3-L1 adipocytes. Mol. Cell. Biol. 21, 5276-5285 (2001).

19. Prigent, M. et al. ARF6 controls post-endocytic recycling through its downstream exocyst complex effector. J. Cell Biol. 163, 1111-1121 (2003).

20. Shteyn, E., Pigati, L. \& Folsch, H. Arf6 regulates AP-1B-dependent sorting in polarized epithelial cells. J. Cell Biol. 194, 873-887 (2011).

21. Vitale, N., Chasserot-Golaz, S. \& Bader, M. F. Regulated secretion in chromaffin cells: an essential role for ARF6-regulated phospholipase D in the late stages of exocytosis. Ann. N. Y. Acad. Sci. 971, 193-200 (2002).

22. Yang, C. Z. \& Mueckler, M. ADP-ribosylation factor 6 (ARF6) defines two insulin-regulated secretory pathways in adipocytes. J. Biol. Chem. 274, 25297-25300 (1999).

23. Karim, F. D. \& Rubin, G. M. Ectopic expression of activated Ras1 induces hyperplastic growth and increased cell death in Drosophila imaginal tissues. Development 125, 1-9 (1998).

24. Halfar, K., Rommel, C., Stocker, H. \& Hafen, E. Ras controls growth, survival and differentiation in the Drosophila eye by different thresholds of MAP kinase activity. Development 128, 1687-1696 (2001).

25. Chabu, C. \& Xu, T. Oncogenic Ras stimulates Eiger/TNF exocytosis to promote growth. Development 141, 4729-4739 (2014).

26. Pagliarini, R. A. \& Xu, T. A genetic screen in Drosophila for metastatic behavior. Science 302, 1227-1231 (2003).

27. Clifford, R. J. \& Schupbach, T. Coordinately and differentially mutable activities of torpedo, the Drosophila melanogaster homolog of the vertebrate EGF receptor gene. Genetics 123, 771-787 (1989).

28. Baker, N. E. Cell proliferation, survival, and death in the Drosophila eye. Semin. Cell Dev. Biol. 12, 499-507 (2001).

29. Johnson Hamlet, M. R. \& Perkins, L. A. Analysis of corkscrew signaling in the Drosophila epidermal growth factor receptor pathway during myogenesis. Genetics 159, 1073-1087 (2001).

30. Rutledge, B. J., Zhang, K., Bier, E., Jan, Y. N. \& Perrimon, N. The Drosophila spitz gene encodes a putative EGF-like growth factor involved in dorsal-ventral axis formation and neurogenesis. Genes Dev. 6, 1503-1517 (1992).

31. Neuman-Silberberg, F. S. \& Schupbach, T. The Drosophila dorsoventral patterning gene gurken produces a dorsally localized RNA and encodes a TGF alpha-like protein. Cell 75, 165-174 (1993).

32. Schweitzer, R., Shaharabany, M., Seger, R. \& Shilo, B. Z. Secreted Spitz triggers the DER signaling pathway and is a limiting component in embryonic ventral ectoderm determination. Genes Dev. 9, 1518-1529 (1995).

33. Tsruya, R. et al. Intracellular trafficking by Star regulates cleavage of the Drosophila EGF receptor ligand Spitz. Genes Dev. 16, 222-234 (2002).

34. Rodrigues, A. B., Werner, E. \& Moses, K. Genetic and biochemical analysis of the role of Egfr in the morphogenetic furrow of the developing Drosophila eye. Development 132, 4697-4707 (2005).

35. Bentley, C. et al. A requirement for wild-type Ras isoforms in mutant KRas-driven signalling and transformation. Biochem. J. 452, 313-320 (2013).

36. James, K. E., Dorman, J. B. \& Berg, C. A. Mosaic analyses reveal the function of Drosophila Ras in embryonic dorsoventral patterning and dorsal follicle cell morphogenesis. Development 129, 2209-2222 (2002).

37. Xu, R. et al. Arf6 regulates EGF-induced internalization of E-cadherin in breast cancer cells. Cancer Cell Int. 15, 11 (2015).

38. Hahn, I. et al. The Drosophila Arf GEF Steppke controls MAPK activation in EGFR signaling. J Cell Sci. 126, 2470-2479 (2013).

39. Marchesin, V., Montagnac, G. \& Chavrier, P. ARF6 promotes the formation of Racl and WAVE-dependent ventral F-actin rosettes in breast cancer cells in response to epidermal growth factor. PLoS ONE 10, e0121747 (2015)

40. Onel, S., Bolke, L. \& Klambt, C. The Drosophila ARF6-GEF Schizo controls commissure formation by regulating Slit. Development 131, 2587-2594 (2004). 
41. Strutt, D. I. \& Mlodzik, M. Hedgehog is an indirect regulator of morphogenetic furrow progression in the Drosophila eye disc. Development 124, 3233-3240 (1997).

42. Tabata, T., Eaton, S. \& Kornberg, T. B. The Drosophila hedgehog gene is expressed specifically in posterior compartment cells and is a target of engrailed regulation. Genes Dev. 6, 2635-2645 (1992).

43. Aza-Blanc, P. \& Kornberg, T. B. Ci: a complex transducer of the hedgehog signal. Trends Genet.: TIG 15, 458-462 (1999).

44. Callejo, A. et al. Dispatched mediates Hedgehog basolateral release to form the long-range morphogenetic gradient in the Drosophila wing disk epithelium. Proc. Natl Acad. Sci. USA 108, 12591-12598 (2011).

45. Mullor, J. L., Calleja, M., Capdevila, J. \& Guerrero, I. Hedgehog activity, independent of decapentaplegic, participates in wing disc patterning. Development 124, 1227-1237 (1997).

46. Rogers, E. M. et al. Pointed regulates an eye-specific transcriptional enhancer in the Drosophila hedgehog gene, which is required for the movement of the morphogenetic furrow. Development 132, 4833-4843 (2005).

47. Lauth, M., Bergstrom, A., Shimokawa, T. \& Toftgard, R. Inhibition of GLI-mediated transcription and tumor cell growth by small-molecule antagonists. Proc. Natl Acad. Sci. USA 104, 8455-8460 (2007).

48. Agyeman, A., Jha, B. K., Mazumdar, T. \& Houghton, J. A. Mode and specificity of binding of the small molecule GANT61 to GLI determines inhibition of GLI-DNA binding. Oncotarget 5, 4492-4503 (2014).

49. Jekely, G. \& Rorth, P. Hrs mediates downregulation of multiple signalling receptors in Drosophila. EMBO Rep. 4, 1163-1168 (2003).

50. Moore, M. J. et al. Erlotinib plus gemcitabine compared with gemcitabine alone in patients with advanced pancreatic cancer: a phase III trial of the National Cancer Institute of Canada Clinical Trials Group. J. Clin. Oncol. 25, 1960-1966 (2007).

51. Ryoo, H. D., Gorenc, T. \& Steller, H. Apoptotic cells can induce compensatory cell proliferation through the JNK and the Wingless signaling pathways. Dev. Cell 7, 491-501 (2004).

52. Peters, P. J. et al. Characterization of coated vesicles that participate in endocytic recycling. Traffic 2, 885-895 (2001).

53. Maranda, B. et al. Intra-endosomal $\mathrm{pH}$-sensitive recruitment of the Arf-nucleotide exchange factor ARNO and Arf6 from cytoplasm to proximal tubule endosomes. J. Biol. Chem. 276, 18540-18550 (2001).

54. Huang, J., Zhou, W., Dong, W. \& Hong, Y. Targeted engineering of the Drosophila genome. Fly (Austin) 3, 274-277 (2009).

55. Huang, J., Zhou, W., Dong, W., Watson, A. M. \& Hong, Y. From the Cover: Directed, efficient, and versatile modifications of the Drosophila genome by genomic engineering. Proc. Natl Acad. Sci. USA 106, 8284-8289 (2009).

56. Lee, T. \& Luo, L. Mosaic analysis with a repressible cell marker (MARCM) for Drosophila neural development. Trends Neurosci. 24, 251-254 (2001).
57. Chabu, C. \& Doe, C. Q. Dap160/intersectin binds and activates aPKC to regulate cell polarity and cell cycle progression. Development 135, 2739-2746 (2008).

\section{Acknowledgements}

We thank T. Schupback (Princeton Univ. NJ), A. Michelson (Harvard Univ. MA), C. Berg (Univ. of Washington, WA), E. Chen (Johns Hopkins Univ. MD), Bloomington stock center, and the Vienna Drosophila RNAi Center for kindly providing reagents. This study was supported in part by grants from the National Basic Research Program of China (MOST2013CB945301) and from NIH/NCI to T.X. C.C. is funded by an NIH/NCI post-doctoral grant (1F32CA142118-01A1). T.X. is a Howard Hughes Medical Institute Investigator

\section{Author contributions}

C.C. and T.X. designed the research. C.C. performed experiments and analysed the data. D.-M.L. performed the mouse and human Arf6 RNAi knockdown experiments. C.C. and T.X. wrote the manuscript.

\section{Additional information}

Supplementary Information accompanies this paper at http://www.nature.com/ naturecommunications

Competing financial interests: The authors declare no competing financial interests.

Reprints and permission information is available online at http://npg.nature.com/ reprintsandpermissions/

How to cite this article: Chabu, C. et al. EGFR/ARF6 regulation of Hh signalling stimulates oncogenic Ras tumour overgrowth. Nat. Commun. 8, 14688 doi: $10.1038 /$ ncomms14688 (2017).

Publisher's note: Springer Nature remains neutral with regard to jurisdictional claims in published maps and institutional affiliations.

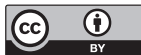

This work is licensed under a Creative Commons Attribution 4.0 International License. The images or other third party material in this article are included in the article's Creative Commons license, unless indicated otherwise in the credit line; if the material is not included under the Creative Commons license, users will need to obtain permission from the license holder to reproduce the material. To view a copy of this license, visit http://creativecommons.org/licenses/by/4.0/

(C) The Author(s) 2017 